\title{
Modelo econométrico basado en la aplicación del costo promedio ponderado de capital para las mipymes del sector metalmecánico en Colombia *
}

\author{
Econometric Model Based on the Application of the Weighted Average Cost of \\ Capital for MSMEs in the Metal-Mechanic Sector in Colombia
}

\author{
Ricardo Fernando Burbano-Delgado (iD \\ Magister en Administración de Empresas, Universidad de Investigación y Desarrollo, \\ Bucaramanga - Colombia, rburbano1@udi.edu.co
}

Youseline Garavito-Hernández

Doctora en Administración y Dirección de Empresas, Universidad de Investigación y Desarrollo, Bucaramanga - Colombia, ygaravito2@udi.edu.co

\begin{abstract}
Cómo citar / How to cite
Burbano-Delgado, R. F., Garavito-Hernández, Y. (2022). Modelo econométrico basado en la aplicación del costo promedio ponderado de capital para las mipymes del sector metalmecánico en Colombia. Revista CEA, v. 8, n. 16, e1974. https://doi.org/10.22430/24223182.1974
\end{abstract}

Recibido: 6 de mayo de 2021

Aceptado: 21 de septiembre de 2021

\begin{abstract}
Resumen
El sector metalmecánico es un componente clave en la dinámica económica colombiana, genera aproximadamente el $15 \%$ de los empleos del país, dinamiza el sector de producción de alimentos, petróleo, fabricación de productos químicos entre otros, además, aporta cerca del $9 \%$ al producto interno bruto (PIB). Este sector se compone, en esencia, por las micro, pequeñas y medianas empresas (mipymes). Esta investigación tuvo como objetivo proponer un modelo econométrico basado en la aplicación del costo promedio ponderado de capital (WACC), para coadyuvar a la toma de decisiones en las mipymes del sector metalmecánico del Área Metropolitana de Bucaramanga. Además, se evaluaron distintos escenarios en función de las tasas de interés de los créditos otorgados a estas. La investigación se realizó a través de un análisis descriptivo, multivariado y de regresión múltiple. Así, teniendo en cuenta el modelo econométrico propuesto en este trabajo y el análisis de
\end{abstract}

\footnotetext{
* Este artículo se deriva del proyecto de investigación «Modelo econométrico basado en la aplicación del WACC para las MiPymes del sector metalmecánico en Colombia» y ha sido financiado con recursos propios.
} 
los distintos escenarios, los resultados muestran que las microempresas del sector alcanzan un mayor WACC ya que al no apalancar financieramente sus activos no aprovechan las bondades que ofrece el escudo fiscal en la reducción de dicho costo. Por otro lado, se encontró que las pequeñas empresas tienen un menor WACC que las medianas empresas sin que ello represente diferencias estadísticas significativas. Se concluye que financiar activos con deuda representa una ventaja directa a la hora del pago de impuestos, lo que se traduce en mayores utilidades para los accionistas, siempre y cuando la política de endeudamiento sea coherente con el desempeño de la empresa. Igualmente, los resultados obtenidos en esta investigación se constituyen en un referente sobre el cual las empresas podrán realizar un análisis estratégico con el fin de identificar hasta qué punto están obteniendo o no valor frente a la dinámica sectorial.

Palabras clave: estructura de capital, WACC, mipymes, sector metalmecánico, regresión lineal múltiple.

Clasificación JEL: M41.

\title{
Highlights
}

- El modelo econométrico basado en el costo del promedio ponderado de capital (WACC) tiene como objetivo coadyuvar a la toma de decisiones de las micro, pequeñas y medianas empresas (mipymes) del sector metalmecánico.

- Las pequeñas empresas tienen un menor costo del promedio ponderado de capital (WACC) que las medianas empresas del sector metalmecánico.

- Las microempresas del sector metalmecánico alcanzan un mayor costo del promedio ponderado de capital (WACC) al no apalancar financieramente sus activos.

- El modelo econométrico basado en el costo del promedio ponderado de capital (WACC) permite a las empresas del sector metalmecánico realizar un análisis estratégico en relación con su valor frente a la dinámica sectorial.

\begin{abstract}
The metal-mechanic sector is a key component in the Colombian economic dynamics. It generates approximately $15 \%$ of the jobs in the country; it stimulates the food, oil, and chemical production sectors, among others; and it also contributes about 9\% to the Gross Domestic Product (GDP). This sector is composed of Micro, Small, and Medium-sized Enterprises (MSMEs). The objective of this study was to propose an econometric model based on the application of the Weighted Average Cost of Capital (WACC) to support the decision-making of MSMEs in the metal-mechanic sector in the Metropolitan Area of Bucaramanga (MAB), Colombia. Furthermore, different scenarios with several interest rates of the loans granted to them were evaluated. This study implemented descriptive multivariate multiple regression analysis. Thus, considering the econometric model proposed here and the analysis of the different scenarios, the results show that the WACC of the microenterprises in this sector is higher because they do not use financial leverage for their assets. Therefore, they do not take advantage of the benefits offered by the tax shield for the reduction of said cost. On the other hand, it was found that small companies have a lower WACC than their medium-sized counterparts, but this difference is not statistically significant. It is concluded that financing assets with debt represents a direct advantage when it comes to paying taxes, which translates into higher profits for shareholders if the debt policy is consistent with the performance of the company.
\end{abstract}


Likewise, the results obtained here constitute a reference point for companies to carry out strategic analyses to establish to what extent they are obtaining value or not in the face of sectoral dynamics.

Keywords: Capital structure, WACC, MSMEs, metal-mechanic sector, multiple linear regression.

JEL classification: M41.

\section{Highlights}

- The econometric model based on the Weighted Average Cost of Capital (WACC) aims to help Micro, Small, and Medium-sized Enterprises (MSMEs) in the metal-mechanic sector to make decisions.

- Small companies in the metal-mechanic sector have a lower WACC than their medium-sized counterparts.

- Micro-enterprises in the metal-mechanic sector have a higher WACC because they do not use financial leverage for their assets.

- The econometric model based on WACC allows companies in the metal-mechanic sector to conduct a strategic analysis of their value in the face of sector dynamics.

\section{INTRODUCCIÓN}

Según el índice de producción real (IPR) (Banco de la República, s.f.), en años recientes, el sector metalmecánico en Colombia ha presentado una tendencia al alza que ha beneficiado a la economía nacional, (el índice de producción real no expresa volúmenes o valores absolutos de producción sino el cambio porcentual en la producción con respecto a un año base que se actualiza periódicamente). Las cifras revelan que el sector proporciona 89079 empleos, lo que representa un $12.55 \%$ del total de la industria manufacturera; respecto al volumen de producción, ocupó el quinto lugar durante el 2018 con una producción valorada en 22.55 billones de pesos (Departamento Administrativo Nacional de Estadística [DANE], 2018). En el departamento de Santander el panorama es similar al nacional; por ejemplo, este sector ocupa el segundo lugar al representar un $16.36 \%$ del total de la industria manufacturera y al tener en sus nóminas aproximadamente a 3162 trabajadores. Con respecto al volumen de producción departamental ocupa el quinto lugar en importancia, alcanzando una participación en los últimos años de 396 mil millones de pesos. Sin embargo, se debe tener en cuenta que el sector petrolero y el sector de alimentos aportan un $76.34 \%$ y un $15.31 \%$, respectivamente, del total de la producción (DANE, 2018).

No obstante, en el ámbito nacional existe un número elevado de fracasos en los primeros años de operaciones en las empresas de este sector, especialmente en las micro, pequeñas y medianas empresas (mipymes), las cuales se caracterizan por un modelo tradicional administrativo (CastroSilva et al., 2019). Las microempresas están conformadas por compañías con un personal no superior a diez trabajadores; las pequeñas tienen entre once y cincuenta trabajadores; y las medianas cuentan entre 51 y doscientos trabajadores (Ley 590, 2000). En términos de financiación, la problemática que atraviesan las mipymes de este sector se debe a que realizan inversiones con recursos propios, lo que conlleva una dificultad para cumplir los compromisos financieros asumidos, generando riesgos morales y volatilidad en retornos futuros de la empresa, presentándose, en consecuencia, problemas 
de liquidez, disminución de la capacidad de crecimiento y su permanencia en el mercado (Rodríguez, 2003; Torralba Flores et al., 2017).

Esta falencia está asociada a una inadecuada estructura financiera sobre la cual se fija explícitamente el costo promedio ponderado de capital (WACC), el cual representa la rentabilidad mínima que se espera de una empresa para crear valor; por lo tanto, no basta tan solo con manejar sólidos conceptos financieros en la dirección de las empresas, hace falta, además, una adecuada estructura financiera (Barrera Lievano et al., 2019). De lo anterior, se propone la siguiente pregunta de investigación: ¿cómo deberá estar trazada la estructura financiera para el apropiado funcionamiento de las mipymes del sector metalmecánico en el Área Metropolitana de Bucaramanga, en Colombia? Para dar respuesta al problema planteado, se propone un modelo econométrico basado en la aplicación del WACC y un análisis de escenarios en función de las tasas de interés de los créditos otorgados a las mipymes, lo cual permitirá coadyuvar a la toma de decisiones de estas empresas.

Así, el presente artículo, en primer lugar, contextualiza los retos a los que se enfrentan las mipymes del sector metalmecánico del Área Metropolitana de Bucaramanga (AMB) en una dinámica económica global. Posteriormente, procede a analizar los elementos básicos de la estructura financiera de cada una de las empresas objeto de estudio con el propósito de calcular el WACC de cada una de ellas y lograr, de esta manera, construir la data sobre la cual se estima el modelo econométrico. Por último, se evalúan distintos escenarios para las mipymes a partir de un análisis longitudinal del comportamiento de las tasas de captación con una ventana temporal de cinco años. Para alcanzar el objetivo de investigación, el artículo se estructura de la siguiente manera: se expone el marco teórico, luego la metodología y posteriormente se presentan los resultados, la discusión y las conclusiones.

\section{MARCO TEÓRICO}

\section{La estructura financiera de una empresa}

La estructura financiera de una empresa está determinada por el capital que invierten los accionistas, y la deuda financiera (Das, 2015), que puede ser a corto o largo plazo, es a través de esta estructura en la que se financian los activos mediante los cuales la empresa desarrolla su actividad (Hall et al., 2004). La estructura financiera está compuesta por: el pasivo, que representa los deberes y obligaciones que la empresa ha contraído con terceros y el patrimonio compuesto por los recursos propios que ha depositado el(los) dueño(s), más las utilidades acumuladas de la actividad en desarrollo y las valoraciones de mercado que a través del tiempo haya adicionado.

La estructura de capital es importante para las organizaciones cuando buscan maximizar su valor (Cornejo Díaz, 2015; Jackova, 2017), involucra la decisión de cuantificar la participación de la deuda, así como el aporte de los socios para financiar los activos. Por tal motivo, Modigliani y Miller (1963) plantean que la decisión de financiamiento del capital es irrelevante en un mercado perfecto; además, demostraron que el impuesto de renta empresarial tiene un efecto positivo en las finanzas de los accionistas. En ese orden de ideas, concluyen que es importante financiar parte de los activos con deuda, con excepción que dicho costo sea excesivo y en cierta medida volátil. En esta misma 
línea, Stiglitz (1974) afirma que las empresas tomarán deuda si existe la seguridad de no generar costos de deuda.

Hoy en día los estudios relacionados con la estructura de capital se desarrollan a través de la herramienta de investigación «velocidad de ajuste de la estructura de capital» (Cornejo Díaz, 2015; Cao y Cui, 2021), es decir, lo que se busca es la versatilidad con que una empresa ajusta su estructura de capital bajo un nivel de apalancamiento requerido o planificado (Hovakimian y Li, 2011; Wojewodzki et al., 2018; Balboa et al., 2017). Bajo esta perspectiva, una empresa presenta una estructura de capital, donde se cuantifica el costo de financiación de sus activos a través del cálculo del WACC, el cual se define como «el promedio aritmético ponderado del costo de los diferentes recursos financieros que utiliza la empresa, después de impuestos, para realizar sus inversiones y operaciones económicas» (Parra Barrios, 2018, p. 8). Su expresión matemática queda definida de la siguiente manera (1).

$$
W A C C=\left(K_{d} * \frac{D}{D+E}\right)\left(1-t_{x}\right)+\left(K_{e} * \frac{E}{D+E}\right)
$$

Donde:

$\mathrm{Kd}$ : Es el costo de la deuda.

D: Deuda de carácter financiero.

E: Equity o capital de inversión de los accionistas.

(1-tx): Escudo fiscal, donde tx representa la tasa impositiva de renta.

Ke: Costo del capital propio.

El capital propio se debe calcular con referencia al mercado, así como el costo de la deuda se calcula una vez se contribuye con el impuesto de renta (Bruner et al., 1998). Esta particularidad hace más difícil el cálculo del costo del capital propio o el rendimiento esperado por los accionistas. Por lo tanto, este costo puede ser calculado mediante distintos métodos como el modelo CAPM (Capital Asset Pricing Model) (Situm, 2021). Este modelo establece que la rentabilidad esperada por los accionistas se expresa mediante la Ecuación (2) (Sharpe, 1964).

$$
K_{e}=r_{f}+\beta_{l} *\left(r_{m}-r_{f}\right)
$$

Donde:

Ke: Costo del capital propio.

rf: Representa una tasa libre de riesgo.

ßI: Beta apalancada que representa básicamente una medida de volatilidad del activo en estudio ante la variabilidad del mercado. La beta se calcula al dividir la covarianza correspondiente a las rentabilidades del mercado y del activo entre la varianza de la rentabilidad del mercado.

rm: Tasa de rentabilidad del mercado.

( $r m$ - rf): Representa la prima de riesgo del mercado.

Para el caso de las empresas que no hacen parte de la bolsa, el cálculo de la beta se realiza a través del método denominado bottom-up (Martínez et al., 2014). Este procedimiento consiste inicialmente en identificar el sector donde se desempeña la empresa, después se procede a calcular las betas sin apalancamiento de esas empresas, posteriormente se obtiene su promedio (alcanzando la beta del 
sector), y finalmente se calculan las betas de las empresas objeto de análisis apalancando la beta del sector al agregar la ratio D/E (deuda divido por equity).

En relación con estudios seminales, se destaca el trabajo de Modigliani y Miller (1958), quienes afirmaron «la estructura de capital no afecta el valor de la empresa»; es decir, los activos deben generar un rendimiento sin importar la estructura de capital que los financia; igualmente aseveran que el costo de capital aumenta a mayor grado de apalancamiento financiero debido a que aumenta el riesgo de los accionistas. Asimismo, se destaca la investigación de Sharpe (1964), quien utiliza el modelo CAPM para calcular el rendimiento que deberían tener las acciones de una empresa donde la expresión de un activo financiero está en función del mercado. Encontrando que unas de las limitantes del modelo residen en la condición de estar cotizando en bolsa, que el mercado no presente clústeres de volatilidad y que las políticas de la empresa en estudio permanezcan constantes en el tiempo.

Por otro lado, Ross (1976) presenta otro enfoque desde la teoría de valoración por arbitraje (APT), la cual establece que el arbitraje garantiza que los activos sin riesgo proporcionan el mismo rendimiento esperado. En tanto, la rentabilidad esperada por el riesgo de una acción queda sujeta a la rentabilidad según el riesgo derivado del factor macroeconómico escogido. Un enfoque más actual es el presentado por Cotner y Fletcher (2000). Estos autores utilizan el proceso de análisis jerárquico de decisiones (AHP) para obtener una estimación de la rentabilidad del riesgo derivado del costo del patrimonio. La metodología implica la identificación inicial de los factores críticos de éxito, proponiendo unos niveles máximos y mínimos, donde un juicio de expertos define el riesgo asociado a cada factor (Fernández y Carabias, 2006). Entre los trabajos recientes se destaca el de Sánchez Segura (2010), el cual está relacionado con la aplicación de estos métodos en Colombia. El autor manifiesta que debido a las dificultades de aplicar modelos propios en empresas de países desarrollados y en economías emergentes, el modelo CAPM representa la forma más idónea a la hora de calcular el costo de capital de cualquier empresa.

\section{El análisis de la regresión lineal}

Según Rodríguez-Lavado (2018), el análisis de regresión lineal es «el conjunto de técnicas usadas para explorar y cuantificar la relación de dependencia entre una variable cuantitativa llamada variable dependiente o respuesta y una o más variables independientes llamadas variables predictoras» (p.7). Esa relación se puede formular mediante la siguiente expresión (3).

$$
Y=\beta_{0}+\beta_{1} X_{1}+\beta_{2} X_{2}+\cdots+\beta_{p} X_{p}+\varepsilon
$$

Donde:

$Y$ : Hace referencia a la variable respuesta.

$\mathrm{X} 1, \mathrm{X} 2, \ldots, \mathrm{Xp}$ : Son las variables explicativas, independientes o regresores.

$\beta 0, \beta 1, \beta 2, \beta p$ : Parámetros estimados que miden el grado de influencia que tienen las variables independientes sobre la variable respuesta.

$\varepsilon$ : Representa el error entre el valor ajustado y observado. 
Frente a la estimación de los parámetros que miden la influencia que tienen las variables explicativas sobre la variable dependiente, esta se realiza bajo el método de mínimos cuadrados ordinarios (MCO) (Faraway, 2015). Ahora bien, si se expresan todos los datos bajo una matriz y suponiendo que se manejan tres variables predictoras se tendría la siguiente forma matricial (ver Figura 1).

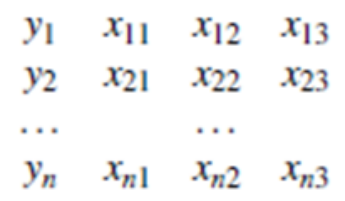

Figura 1. Forma matricial

Figure 1. Matrix form

Fuente: elaboración propia.

Donde $\mathrm{n}$ es el número de observaciones que son objeto de análisis; por otro lado, este arreglo matricial se puede expresar por medio de la siguiente matriz / vector (4).

$$
y=X \beta+\varepsilon
$$

Además, si la mejor estimación de $\beta$ es la que minimiza la suma de los cuadrados errores, entonces se tendría la siguiente expresión (5).

$$
\sum_{i=1}^{n} \varepsilon_{i}^{2}=(y-X \beta)^{T}(y-X \beta)
$$

Se trata de obtener el valor de $\beta$ que minimice el error, este valor se obtiene diferenciando respecto a $\beta$ e igualando a cero (Tusell, 2011). El ajuste de los datos se evidencia, básicamente, por el valor de las diferencias entre el valor real y el ajustado elevadas al cuadrado; esta particularidad permite «castigar» las diferencias, evitando valores negativos. Asimismo, unas diferencias elevadas hacen que la recta no represente los datos; por el contrario, pequeñas diferencias indican un buen ajuste. Es importante advertir que los errores deben tener una distribución normal, ser independientes e idénticamente distribuidos con varianza constante y media cero, es decir, $\varepsilon \sim N\left(0, \sigma^{2} I\right)$.

\section{METODOLOGÍA}

La investigación es cuantitativa y explicativa al proponer un modelo econométrico basado en la aplicación del WACC, con lo cual se explicará la estructura de capital de las empresas del sector metalmecánico en diferentes escenarios. Como fuente de información se utilizó la base de datos del DANE y de la Dirección de Impuestos y Aduanas Nacionales (DIAN). Además, se recurrió al software especializado EMIS University, que ofrece información contable de más de tres millones de empresas de «mercados emergentes» y se consultaron diversos portales financieros con el fin de construir las variables de la investigación.

En relación con la muestra, teniendo en cuenta la base de datos del DANE, se incluyeron en el estudio todos los establecimientos industriales reconocidos dentro de la Clasificación Industrial Internacional Uniforme - Revisión 4, adaptada para Colombia, en la cual se encontró un total de 49 empresas registradas en el Área Metropolitana de Bucaramanga; sin embargo, la base de datos proporcionada 
por el software EMIS University arrojó un tamaño de 106 empresas, donde posteriormente, al realizarle un proceso de depuración, se obtuvieron 51 empresas. Ver Tabla 1.

Tabla 1. Universo de las empresas del sector metalmecánico en el AMB de acuerdo con el instrumento utilizado

Table 1. Universe of companies in the metal-mechanic sector in the MAB according to the instrument

\begin{tabular}{ccccc}
\hline Instrumento & Micro & Pequeña & Mediana & Total \\
\hline DANE & 12 & 19 & 18 & 49 \\
\hline EMIS University & 24 & 14 & 13 & 51 \\
\hline
\end{tabular}

La validez del constructo y de contenido para los distintos instrumentos contó con la ventaja de que la Encuesta Anual de Manufactura 2018 tiene un proceso ya establecido por los lineamientos del DANE, mientras que el instrumento del EMIS University se ajusta a la teoría contable soportada por los organismos de vigilancia y control estatales. A todos los instrumentos se les realizó un análisis de datos faltantes (NAs), encontrándose que en el software EMIS University existen varios campos «NA» pertenecientes a empresas que no han reportado sus estados financieros, y que por lo tanto fueron eliminadas del análisis. Por otro lado, se encontraron valores atípicos que fueron tratados específicamente mediante el análisis univariado, empleando técnicas gráficas como el boxplot. Igualmente, a todos los instrumentos se les analizó sus unidades de medida, formatos, espacio temporal, entre otros.

Con respecto al desarrollo metodológico de esta investigación, se realizó, en primer lugar, un análisis descriptivo que permitió la caracterización del sector, ayudó a identificar la presencia de datos anómalos y le dio validez al modelo de regresión lineal múltiple. En segundo lugar, se calculó el WACC, para tener claro los componentes de su estructura, así, al revisar la Ecuación (1), se muestran tres variables que son «independientes» del comportamiento financiero: costo de la deuda, escudo fiscal y la rentabilidad exigida por los accionistas. El cálculo de estas variables depende principalmente del aporte de la información de las empresas (costo de la deuda); sin embargo, existen limitaciones en las que fue necesario acudir a estudios como el desarrollado por la Gran Encuesta Pyme 2018, realizada por la Asociación Nacional de Instituciones Financieras (ANIF).

A continuación, se realizó un modelo de regresión lineal múltiple utilizando las variables significativas enunciadas en el marco teórico en la Ecuación (1) para calcular el WACC. Esto conlleva a un cálculo más sencillo y al alcance de cualquier empresa formalizada. Además de su practicidad, la interpretación de las variables se hace más evidente, ya que en este caso muestra qué implicación tiene en el WACC el tamaño de la empresa y cómo lo afecta concretamente la política de financiamiento asumida por la empresa, así como las tasas de interés del mercado. En resumen, el modelo de regresión lineal múltiple permite incorporar la relación existente entre el tamaño de la empresa, la política de endeudamiento y la coyuntura macroeconómica en el valor del WACC.

Finalmente, se realizó un análisis de distintos escenarios que busca encontrar un posible comportamiento del sector metalmecánico de acuerdo con el tamaño de la empresa; gracias a este análisis se puede guiar a los empresarios en la definición de una política de endeudamiento de acuerdo con la coyuntura económica por la que atraviesan lo que permite reducir el riesgo de insolvencia. Igualmente, dentro de cada escenario planteado se muestra la vulnerabilidad o fortaleza 
del sector, lo que facilita la formulación de políticas públicas más apropiadas de acuerdo con el contexto macroeconómico y sus perspectivas.

\section{RESULTADOS}

\section{Caracterización del sector metalmecánico}

El DANE señala que las micro, pequeñas y medianas empresas en Colombia representan el $35 \%$ del PIB, aportando a la producción nacional el $90 \%$, y más del $80 \%$ del empleo del país; un crecimiento lento, pero significativo, según reporte que presentó la ANIF en el 2018, ya que en este informe se evidencia que las mipymes representan el 96\% del total de las empresas del país, la empleabilidad alcanza el $63 \%$ y contribuyen con el $25 \%$ del PIB nacional (ANIF, 2018). Según (Confecámaras, 2019), solo en el primer trimestre del año 2019 se crearon aproximadamente 96914 empresas, un 9.7\% más que el mismo periodo del año anterior. De estas empresas creadas, el $77.8 \%$ corresponden a «personas naturales», y el restante $22.2 \%$ correspondieron a «personas jurídicas». Ver Figura 2.

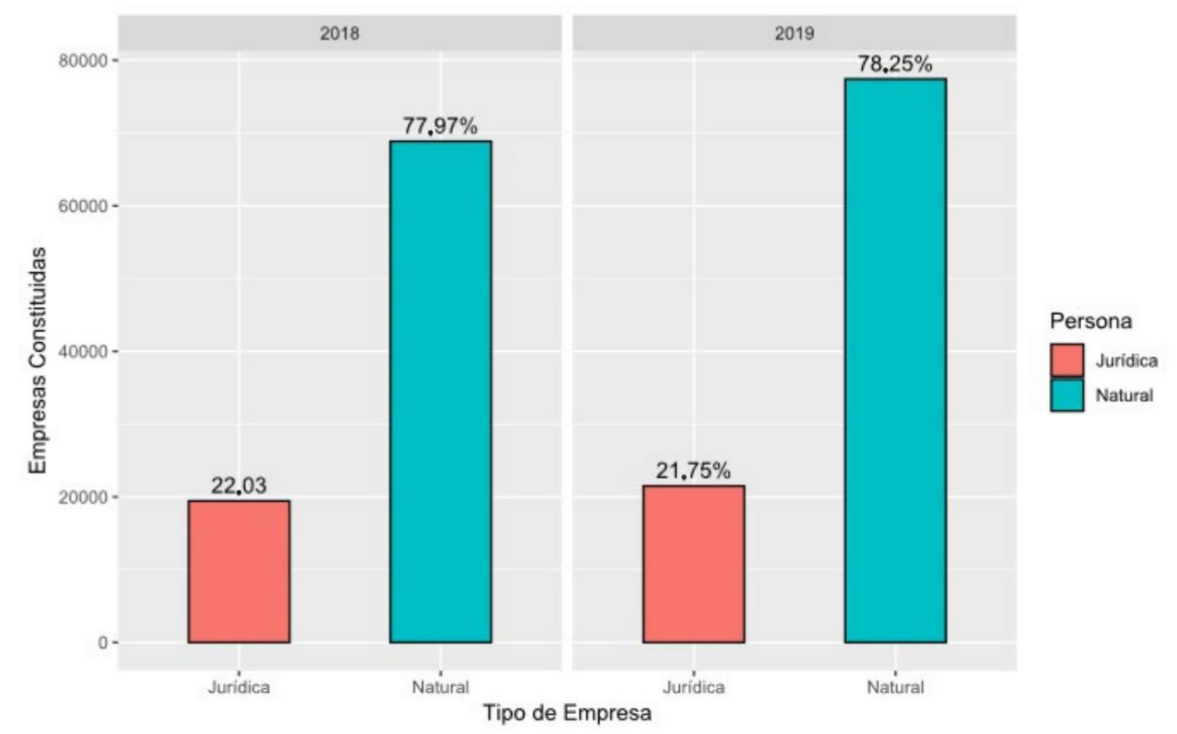

Figura 2. Unidades productivas creadas según organización jurídica durante el primer trimestre de los años 2018 y 2019

Figure 2. Businesses created during the first quarter of 2018 and 2019 classified by type of company Fuente: elaboración propia adaptado de (Confecámaras, 2019).

De acuerdo con los datos proporcionados por la Encuesta Anual Manufacturera - EAM (2018), realizada por el DANE, frente a las distintas formas de contratación, se encontró que aproximadamente el $81.63 \%$ de los empleos permanentes son generados por las empresas medianas, el $13.29 \%$, por las pequeñas, y un $5.08 \%$ lo generan las microempresas.

Frente a los activos fijos, las microempresas del sector metalmecánico alcanzan un monto de \$371 millones, además, el $50 \%$ de estas empresas tienen activos fijos que oscilan entre los $\$ 98$ millones hasta los $\$ 383$ millones. Por su parte, la pequeña empresa muestra que el $50 \%$ tienen un monto de activos fijos entre los $\$ 628$ millones hasta los $\$ 2035$ millones, mientras que en promedio la mediana empresa alcanza los $\$ 9724$ millones en activos fijos. 
En lo referente al pago de arriendo de maquinaria e inmuebles, la encuesta arroja que el $8.3 \%$ de las microempresas arriendan maquinaria, la pequeña empresa un $47.36 \%$ y le mediana empresa un $72.23 \%$. Para el caso del pago de arriendo por el uso de inmuebles, las tendencias son similares a las del pago de arriendo por maquinaria.

Respecto al costo de mantenimiento de equipos y el pago de pólizas de seguro, las microempresas asignan en promedio el $2.34 \%$ del valor de los activos fijos a los costos de mantenimiento, la pequeña empresa un $1.83 \%$ y la mediana empresa un $2.77 \%$. Los porcentajes son similares, por lo que las empresas del sector metalmecánico están asumiendo costos de mantenimiento (en proporción al monto de los activos fijos) similares.

Por otro lado, el costo de materia prima de las empresas encuestadas durante el año 2018 ascendió a los 184.52 mil millones de pesos, mientras que los ingresos por ventas fueron de 391.65 mil millones de pesos, lo que significa que, en promedio, las empresas del sector metalmecánico invirtieron el $47.11 \%$ de sus ingresos en la compra de materia prima.

Es importante destacar que un gran porcentaje de las empresas del sector metalmecánico importa su materia prima (no necesariamente su totalidad); por tamaño, la mediana empresa importa el $38.88 \%$ del costo total de materia prima, la pequeña empresa importa el $15.78 \%$ y la microempresa no importa materia prima.

\section{Estimación del modelo de regresión múltiple para las mipymes del sector metalmecánico en el AMB}

\section{Análisis Descriptivo}

Analizar previamente la data utilizada para estimar el modelo es un paso necesario que ayuda a identificar algunos lineamientos a la hora de escoger el modelo final. En ese sentido vale la pena describir las variables involucradas en el modelo estimado (ver Tabla 2).

Tabla 2. Variables involucradas en el modelo de regresión estimado Table 2. Variables involved in the estimated regression model

\begin{tabular}{ccc}
\hline Variable & Tipo & Descripción \\
\hline WACC & Continua & Variable dependiente \\
\hline Clasificación & Ordinal & Clasifica las empresas de acuerdo con su tamaño \\
\hline Kd & Continua & Costo de la deuda \\
\hline E/E+D & Continua & Porcentaje de equity en la estructura del capital \\
2. El costo de la deuda se expresa en dos valores de acuerdo con los resultados de la encuesta Gran Encuesta Pymes 2018 \\
\end{tabular}

Por su parte, la Tabla 3 muestra algunos estadísticos de resumen de las variables involucradas en el modelo estimado. 
Tabla 3. Principales estadísticos de resumen de las variables involucradas en el modelo obtenido Table 3. Main summary statistics of the variables involved in the model obtained

\begin{tabular}{ccccccc}
\hline Variable & Mínimo & 1mer cuantil & Mediana & Media & 3cer cuantil & Máximo \\
\hline WACC & 0.1206 & 0.138 & 0.1491 & 0.1433 & 0.1499 & 0.1499 \\
\hline Kd & 0.0854 & 0.0854 & 0.1254 & 0.1113 & 0.1254 & 0.1254 \\
\hline E/E+D & 0.3251 & 0.643 & 0.9468 & 0.8201 & 1 & 1 \\
\hline & Fuente: elaboración propia adaptado de la Gran Encuesta pyme (ANIF, 2018).
\end{tabular}

Respecto a la clasificación, la muestra consta de 51 empresas de las cuales el $47.05 \%$ son microempresas, el $27.45 \%$ son pequeñas empresas y el restante, $25.5 \%$, son medianas empresas. De igual manera, la microempresa, al no contar con deuda en su estructura de capital, hace que su WACC sea constante y que le sea indiferente el costo de la deuda lo que conlleva a la creación de sesgos en las variables involucradas en el modelo estimado. Esta característica hace que el modelo presente dificultades en el cumplimiento de sus supuestos; en esa medida, se excluyeron las microempresas en su estimación.

La Figura 3 muestra que, precisamente, el WACC para la microempresa se comporta como una línea al ser una constante; además, en comparación con las pymes, es evidente que su WACC es significativamente mayor. Es importante evidenciar el efecto del apalancamiento financiero en el WACC, pues las microempresas, al no financiar sus activos con deuda, presentan un costo mayor que las pymes. Por otro lado, no parecen existir diferencias significativas entre el WACC de las pequeñas y medianas empresas, aunque estas últimas tienen una mediana mayor que las primeras. Otro aspecto que se puede resaltar son las distribuciones, en donde, para el caso de la pequeña empresa, los cuantiles 2 y 3 (que albergan el $50 \%$ de los datos), presentan amplia dispersión en comparación con la mediana empresa, es decir, las pequeñas empresas, en términos generales, tienen distintos comportamientos en su estructura de capital, contrario a las medianas empresas que tienden a ser más homogéneas, aunque tienen una gran dispersión entre el primer y segundo cuantil (25\% de los datos).

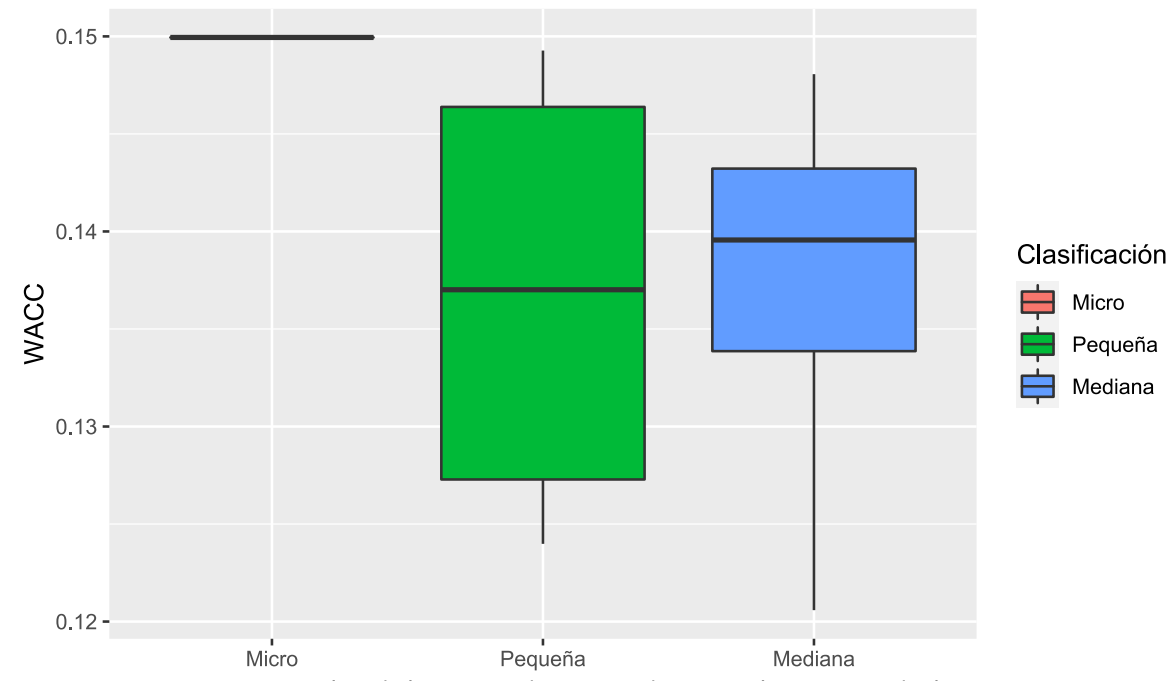

Figura 3. Boxplot del WACC de acuerdo con el tamaño de la empresa

Figure 3. Boxplots of the WACC of companies classified by size

Fuente: elaboración propia. 


\section{Estimación del modelo}

El análisis descriptivo desarrollado previamente permitió establecer un primer contacto con los datos, descartando, inicialmente, la presencia de datos faltantes, outliers, problemas de unidades, entre otros. Asimismo, se logró descartar la microempresa como variable predictora al mantener un valor WACC constante; también se identificó la distribución de las variables involucradas con el fin de establecer posibles transformaciones para el cumplimiento de los supuestos del modelo. Los parámetros del modelo se estimaron con el programa $\mathrm{R}$ Statistics 4.0 .0 a partir del método de «mínimos cuadrados ordinarios» (el mejor método de estimación siempre y cuando los errores no estén correlacionados y tengan la misma varianza) (Valverde y Valverde, 2006). Los resultados se muestran en la Figura 4.

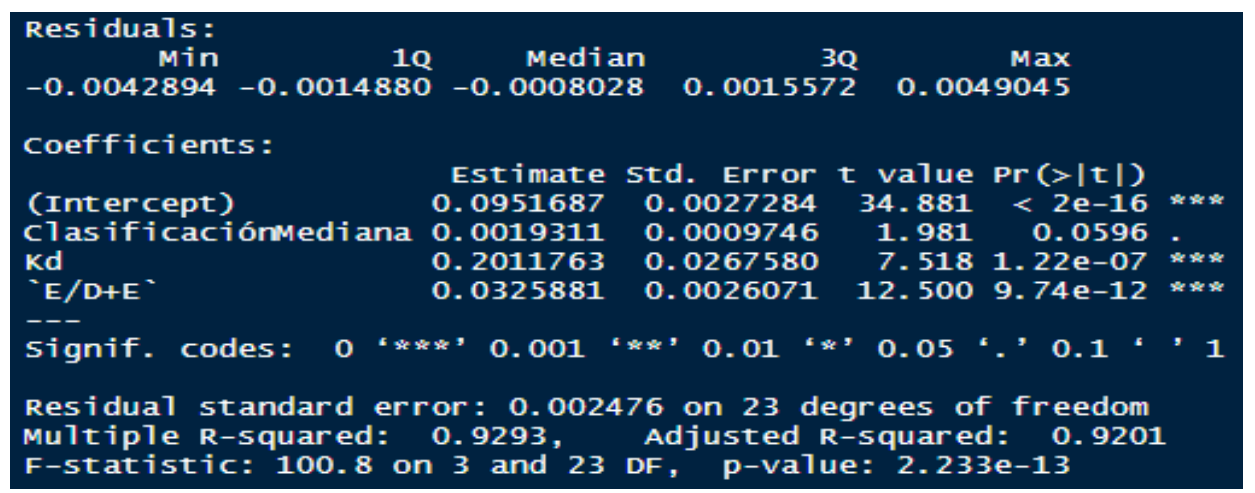

Figura 4. Resultados de la estimación del modelo econométrico

Figure 4. Results of the estimation of the econometric model

Fuente: elaboración propia.

Los resultados muestran que el modelo obtenido estará compuesto por tres covariables más el intercepto, es decir, costo de capital, la clasificación de la mediana empresa (variable dummy [toma valores 0 y 1 , si su valor es cero se hace referencia a una empresa clasificada como pequeña; por el contrario, si el valor es 1 se hace referencia a una empresa mediana]), el porcentaje de equity dentro de la estructura financiera y el intercepto. Es importante que el porcentaje de deuda dentro de la estructura financiera implícitamente esté anidada en el porcentaje de equity, ya que es un complemento de esta, de ahí que no se incluyera en el modelo. Con el fin de establecer mejor claridad frente a la estimación de los parámetros se calcularon adicionalmente sus intervalos de confianza. Ver Figura 5.

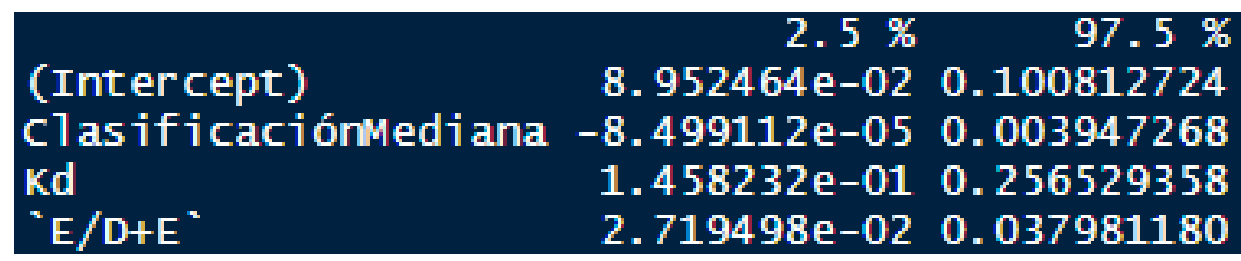

Figura 5. Intervalos de confianza de los parámetros del modelo

Figure 5. Confidence intervals of the model parameters Fuente: elaboración propia. 
El intervalo de confianza del $95 \%$ para la clasificación de mediana empresa apenas incluye el cero. Se podría decir que esta variable no aporta al modelo, sin embargo, se decidió incorporarla al tratarse de una variable «dummy» que, al momento de un análisis, ayuda a establecer las características de la empresa de acuerdo con su tamaño. De la Figura 5 se concluye lo siguiente.

1. Las variables del modelo resultaron significativas al $5 \%$, incluido el intercepto.

2. La bondad de ajuste representada por el coeficiente de determinación o el R2 ajustado es igual a 0.9201 . Dado que: $0 \leqslant R 2 \leqslant 1$, entonces la recta de regresión explica un $92.01 \%$ la variabilidad del modelo.

3. La mediana de los errores se acerca a cero, siendo un referente optimista en la medida que se espera que los errores tengan una distribución normal.

El modelo econométrico obtenido presenta la siguiente estructura (6):

$$
Y=0.0951+0.0019 X_{1}+0.2011 X_{2}+0.0325 X_{3}+\epsilon
$$

Donde:

Y: Representa el valor del WACC en decimales.

X1: Asume dos valores: 1 si la empresa es clasificada como mediana y 0 cuando es clasificada como pequeña.

X2: Representa el costo de la deuda.

X3: Representa el porcentaje del equity dentro de la estructura financiera expresado en decimales. $€$ : Representa el error entre el valor ajustado y observado.

La interpretación de los parámetros sería la siguiente.

1. El intercepto del modelo representa el valor base del WACC que se puede encontrar en el sector metalmecánico en al Área Metropolitana de Bucaramanga. Su valor es importante, ya que está indicando específicamente que las empresas del sector tendrán un WACC superior o que este es el punto de partida sobre el cual se deberá establecer la estructura de deuda de la empresa.

2. Al mantener constantes el costo de la deuda y el porcentaje de equity es claro que el WACC es mayor para medianas empresas. Esto se debe a que la variable dummy es 1 si es mediana empresa y 0 si es pequeña empresa, por lo tanto, al mantener las demás variables constantes se espera que el WACC de la mediana empresa esté un $0.0019 \%$ por encima del que presente la pequeña empresa.

3. Al incrementar el costo de la deuda en un $1 \%$ manteniendo constantes las demás variables, el WACC aumenta en un $0.2011 \%$.

4. Al incrementar el porcentaje de equity sobre en un $1 \%$ manteniendo constantes las demás variables, el WACC aumenta en un $0.0325 \%$.

Los puntos 3 y 4 ayudan a configurar el efecto que produce en el WACC la tasa de interés y la estructura financiera, lo que se muestra es que los cambios en las tasas de interés perturban en mayor medida el aumento de equity dentro de dicha estructura. 


\section{Análisis de escenarios del costo de capital}

Los distintos escenarios a los que se puede enfrentar el empresario del sector metalmecánico en el cálculo del WACC debe tener en cuenta el costo de la deuda, el grado de volatilidad del activo en el mercado y la misma estructura de capital; sin embargo, el modelo obtenido tiene en cuenta únicamente el costo de la deuda, la clasificación de la empresa y la estructura financiera. En esa medida, los distintos escenarios que se le pueden presentar al empresario del sector únicamente estarán formados por la variabilidad de las tasas de captación y las políticas de las entidades bancarias. Conviene entonces analizar el comportamiento del Depósito a Término Fijo (DTF) en los últimos cinco años, así como los resultados de la encuesta ANIF que muestran, básicamente, que en un $60 \%$ de los casos los préstamos se encuentran 4 puntos por encima de la DTF.

La Figura 6 muestra la serie semanal de las cotizaciones del DTF en Colombia a partir del 1 de enero del 2014 hasta el 31 de diciembre del 2018, en ella se puede apreciar que no presenta media ni varianza constante por lo que no es estacionaria, tampoco se aprecia un componente cíclico ni estacional. Debido a lo anterior su tendencia no es homogénea; por ejemplo, inicialmente (año 2014) presenta una breve tendencia a la baja, posteriormente entra en un periodo aparentemente estacionario hasta finales del 2015, a partir de ese momento el DTF presentó una clara tendencia al alza que alcanzó su punto máximo de $7.59 \%$ en el mes de julio del año 2016. Finalmente, después de alcanzar el máximo nivel el DTF, presentó una clara tendencia a la baja hasta finales del año 2018, momento en el cual presenta estabilidad.

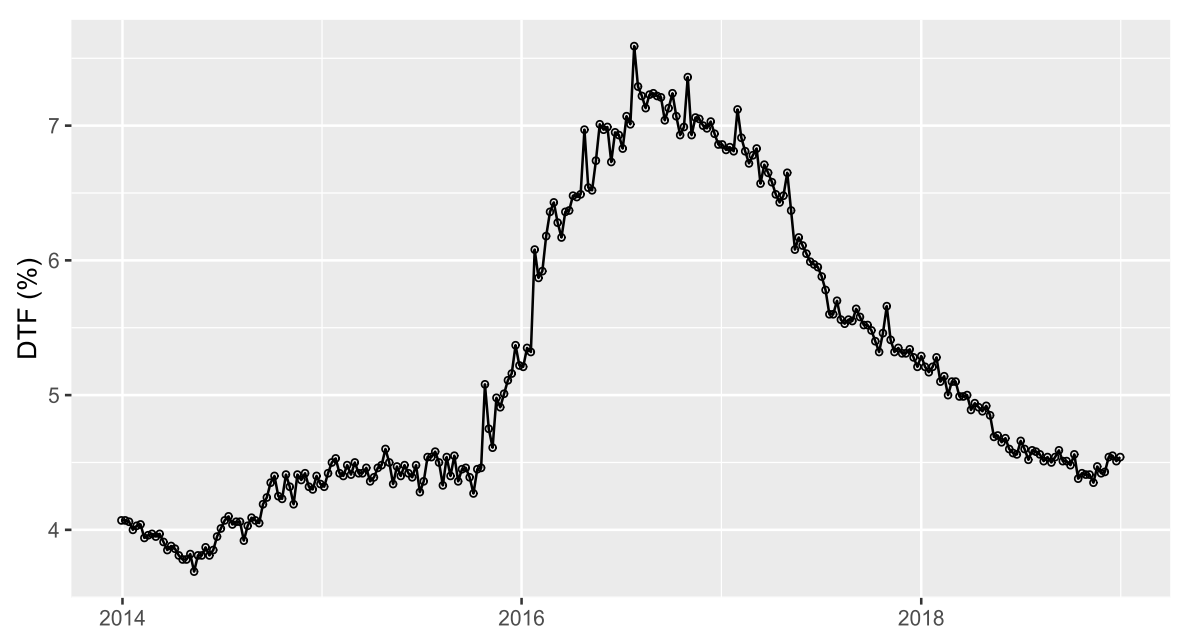

Figura 6. Serie semanal de las tasas de captación en Colombia. Adaptado de Banco de la República Figure 6. Weekly series of deposit rates in Colombia. Adapted from Banco de la República Fuente: elaboración propia.

La Figura 7 muestra el histograma y el gráfico boxplot del DTF; en el primero se puede apreciar un valor modal de aproximadamente $4.5 \%$, además, trata de presentar un sesgo positivo sin llegar a mostrar una distribución simétrica. El boxplot muestra que no existen «outliers», sin embargo, se evidencia dispersión de datos por encima de la mediana, lo que significa que los valores del DTF, en su gran mayoría, tratan de concentrarse por debajo del $5 \%$. 

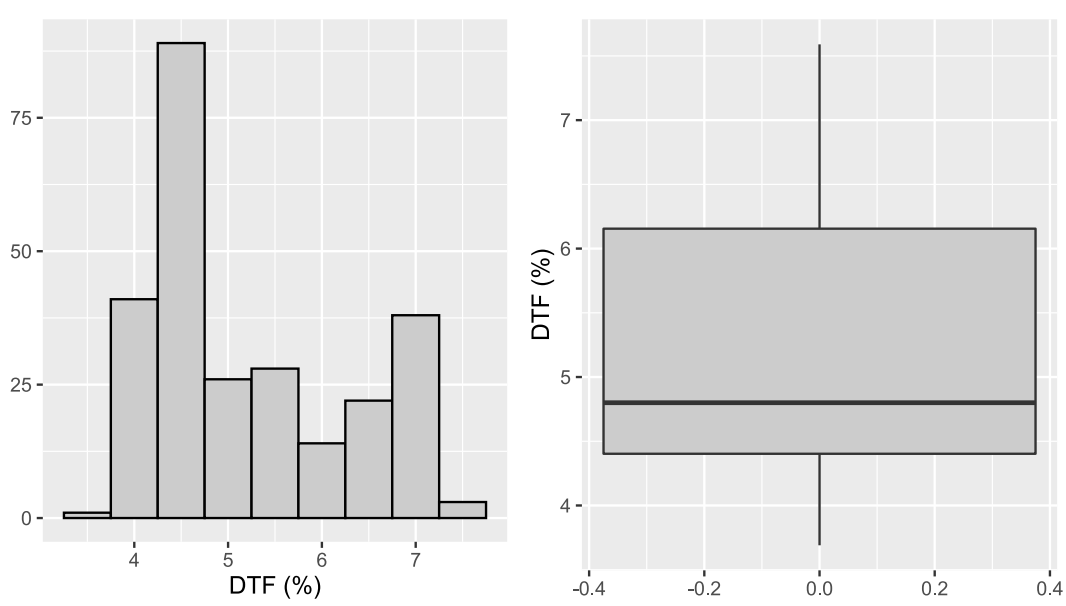

Figura 7. Distribución del DTF y sus cuartiles

Figure 7. Distribution of TD rate and its quartiles

Fuente: elaboración propia.

El análisis descriptivo ayudó a configurar tres escenarios posibles con los cuales se busca establecer el comportamiento del sector metalmecánico en términos del costo promedio ponderado de capital. Igualmente es necesario involucrar del porcentaje del equity que sirva como referencia en cada uno de los escenarios, para ello se tomó la mediana de la distribución de los datos por cada tamaño. Ver Tabla 4.

Tabla 4. Principales medidas de localización del porcentaje de equity dentro de la estructura financiera de las pymes

Table 4. Main measures of location of the equity percentage in the financial structure of the SMEs

\begin{tabular}{lcccccc}
\hline Variable & Mínimo & 1mer cuantil & Mediana & Media & 3cer cuantil & Máximo \\
\hline Mediana & 0.3251 & 0.5904 & 0.6445 & 0.6753 & 0.7774 & 0.9307 \\
\hline Pequeña & 0.4033 & 0.4791 & 0.7027 & 0.6864 & 0.8829 & 0.9598 \\
\hline \multicolumn{7}{c}{ Fuente: elaboración propia. }
\end{tabular}

Los resultados de la Tabla 4 muestran, para el caso de la mediana empresa, una gran concentración de datos en torno al primer y tercer cuantil, reflejando, además, un mayor porcentaje de endeudamiento en comparación con la pequeña empresa. En esa medida los escenarios serían los siguientes:

\section{Escenario pesimista}

La Figura 6 muestra que la tendencia decreciente del DTF parece haber llegado a su fin, se espera que no se avecine un cambio drástico de tendencia, por lo tanto, se tomará el valor del DTF representado en el tercer cuantil igual al $6.155 \%$.

\section{Escenario optimista}

La gran mayoría de las tasas del DTF están por debajo del 6\%, sin embargo, la Figura 6 muestra un cambio de tendencia que hace pensar que dichas tasas no alcanzarán el nivel mínimo registrado, por lo tanto, se tomará el primer cuantil igual a $4.402 \%$. 


\section{Escenario probable}

Siguiendo la lógica se esperaría tomar la mediada como referente, sin embargo, esta marca un sesgo optimista teniendo en cuenta que probablemente aumenten las tasas del DTF; en esa medida es más representativo tomar la media como el escenario más probable, la cual representa el 5.21\%.

\section{Escenarios por tamaño de empresa}

Los escenarios, previamente descritos, se aplicarán no sobre una empresa o varias empresas en concreto, sino una respuesta media del sector metalmecánico segmentado por el tamaño de empresa. Lo que se requiere inicialmente es que las empresas puedan comparar sus resultados con los esperados en el sector, logrando de esta manera establecer metas financieras en el intervalo de tiempo que sus directivos consideren pertinente.

\section{Escenario para la microempresa}

Durante el proceso de investigación se establecieron distintas características que diferencian este tipo de empresas con las pymes, una que sobresale es que las microempresas del sector metalmecánico en el AMB, financian sus activos (por lo menos oficialmente) únicamente con el capital de sus accionistas. Esta característica hace que el WACC sea constante e igual a la rentabilidad esperada por los accionistas desde el punto de vista del sector, independientemente de la microempresa que se analice. Si se observa la Ecuación (7), que busca el cálculo de la beta para la empresa, se encuentra que está en función de la deuda; en ese sentido, si la empresa no apalanca sus activos con deuda, su beta, como empresa, es igual a la beta del sector.

$$
\begin{gathered}
\beta_{l}=\beta_{s}\left(1+\left(1-t^{*}\right) \frac{D}{E}\right) \\
\beta_{l}=\beta_{s}\left(1+\left(1-t^{*}\right) \frac{0}{E}\right) \\
\beta_{l}=\beta_{s}
\end{gathered}
$$

En consecuencia, de acuerdo con la Ecuación (2), la rentabilidad esperada por los inversionistas es igual para cada uno de los microempresarios, ya que tanto la tasa del rendimiento del mercado $r_{m}$ como la tasa libre de riesgo $r_{f}$ son constantes (8).

$$
\begin{aligned}
& K_{e}=r_{f}+\beta_{l} *\left(r_{m}-r_{f}\right) \\
& K_{e}=r_{f}+\beta_{s} *\left(r_{m}-r_{f}\right)
\end{aligned}
$$

Una vez analizadas las Ecuaciones (2) y (7) se procede a verificar los resultados de la Ecuación 1, recordando que la deuda es igual a cero, y que el rendimiento esperado por los inversionistas es constante, con lo cual se obtienen los siguientes resultados (9).

$$
\begin{gathered}
\text { WACC }=\left(K_{d} * \frac{D}{D+E}\right)\left(1-t_{x}\right)+\left(K_{e} * \frac{E}{D+E}\right) \\
W A C C=\left(K_{d} * \frac{0}{0+E}\right)\left(1-t_{x}\right)+\left(K_{e} * \frac{E}{0+E}\right)
\end{gathered}
$$




$$
\begin{gathered}
W A C C=\left(K_{d} * 0\right)\left(1-t_{x}\right)+\left(K_{e} * 1\right) \\
W A C C=K_{e}
\end{gathered}
$$

Como se advirtió previamente en la estimación del modelo de regresión múltiple, el WACC para las microempresas es constante e igual a $14.99 \%$, significativamente mayor que el calculado en las pymes.

\section{Escenario para la pequeña empresa}

Tomando como base la mediana de su porcentaje de equity (70.27\%) dentro de la estructura de capital, los escenarios serían los siguientes.

Optimista: en este escenario, con un costo financiero del $4.402 \%$ EA, se esperarían los siguientes resultados (10).

$$
\begin{gathered}
Y=0.0951+0.0019(0)+0.2011(0.0402)+0.0325(0.7027) \\
Y=12.69 \%
\end{gathered}
$$

Con un intervalo de confianza del $95 \%$ cuyo límite superior es de $13.02 \%$ e inferior: $12.37 \%$.

Pesimista: en este escenario con un costo financiero del 6.155\% EA, se esperarían los siguientes resultados (11).

$$
\begin{gathered}
Y=0.0951+0.0019(0)+0,2011(0,06155)+0,0325(0,7027) \\
Y=13,04 \%
\end{gathered}
$$

Con un intervalo de confianza del 95\%, cuyo límite superior es de $13.28 \%$ e inferior: $12.8 \%$.

Probable: en este escenario, con un costo financiero del $5.21 \%$ EA, se esperarían los siguientes resultados (12).

$$
\begin{gathered}
Y=0,0951+0,0019(0)+0,2011(0,0521)+0,0325(0,7027) \\
Y=12,85 \%
\end{gathered}
$$

Con un intervalo de confianza del 95\% cuyo límite superior es de $13.24 \%$ e inferior: $12.57 \%$.

\section{Escenario para la mediana empresa}

Tomando como base la mediana de su porcentaje de equity (64.45\%) dentro de la estructura de capital, los escenarios serían los siguientes. 
Optimista: en este escenario, con un costo financiero del $4.402 \%$ EA, se esperarían los siguientes resultados (13).

$$
\begin{gathered}
Y=0.0951+0.0019(1)+0.2011(0.0402)+0.0325(0.6445) \\
Y=12.88 \%
\end{gathered}
$$

Con un intervalo de confianza del $95 \%$, cuyo límite superior es de $13.24 \%$ e inferior: $12.53 \%$.

Pesimista: en este escenario, con un costo financiero del $6.155 \%$ EA, se esperarían los siguientes resultados (14).

$$
\begin{gathered}
Y=0.0951+0.0019(1)+0.2011(0.06155)+0.0325(0.6445) \\
Y=13.23 \%
\end{gathered}
$$

Con un intervalo de confianza del $95 \%$, cuyo límite superior es de $13.51 \%$ e inferior: $12.96 \%$.

Probable: en este escenario, con un costo financiero del 5.21\% EA, se esperarían los siguientes resultados (15).

$$
\begin{gathered}
Y=0.0951+0.0019(1)+0.2011(0.0521)+0.0325(0.6445) \\
Y=13.04 \%
\end{gathered}
$$

Con un intervalo de confianza del 95\%, cuyo límite superior es de $13.36 \%$ e inferior: $12.73 \%$.

Los resultados obtenidos han pasado por un proceso de validez y confiabilidad apropiados, ya que se fundaron en información proveniente de instituciones reconocidas oficialmente, además, el procesamiento de la información se sometió a las metodologías propuestas en el marco teórico; en ese sentido, para el cálculo del WACC, se siguieron las pautas del método bottom-up; igualmente, la estimación del modelo se validó mediante el proceso de diagnóstico, mientras que los escenarios de evaluación se establecieron bajo análisis de series de tiempo con el apoyo de la estadística descriptiva. Sin duda, los resultados obtenidos en la presente investigación son pertinentes para la toma de decisiones de las mipymes del sector metalmecánico (empresas del AMB), ya que la información obtenida es una expresión válida de sus características particulares y de la dinámica macroeconómica del país, evitando, de esta manera, el sesgo de factores externos subjetivos.

\section{DISCUSIÓN}

Actualmente es reconocido que una de las causas que llevan al fracaso empresarial de cualquier empresa es el mal manejo financiero y un limitado acceso al crédito; estas falencias están relacionadas con una inadecuada estructura financiera sobre la cual se fije explícitamente el costo promedio ponderado de capital (Torralba Flores et al., 2017). En este orden de ideas, para dar solución a esta problemática, se destacan estudios en el caso colombiano, como el de Bonilla Bonilla 
(2016), quien diseño un modelo para estimar el WACC), mediante betas contables, para las pymes del sector industria caucho y plástico de la ciudad de Bogotá. Los resultados muestran que es posible construir modelos de apoyo a la gestión financiera de las empresas a partir del CAPM y del WACC.

Igualmente, Pachón Palacios (2013) planteó una metodología para calcular el costo de los recursos de los accionistas sin apalancamiento financiero a través de la estimación de la prima de riesgo con una metodología multicriterio. Así, teniendo en cuenta la ecuación definida por el CAPM, y al aplicar el estudio a treinta empresas colombianas, obtuvieron como resultado que la mínima rentabilidad o costo de los recursos propios sin apalancamiento financiero que debe requerir un inversionista al destinar sus recursos es del $10.7 \%$, que corresponde a una prima de riesgo de $2.50 \%$.

Con respecto al valor agregado de esta investigación, en primer lugar, se destaca la profundidad del estudio, ya que, partiendo de una serie de variables independientes, se formuló un modelo econométrico que identificara el estado de la estructura de capital para las empresas del sector metalmecánico del Área Metropolitana de Bucaramanga (Colombia). Además, las implicaciones prácticas del estudio residen básicamente en que se puede llegar a ajustar una estructura financiera adecuada para las empresas del sector, siempre y cuando tengan presentes las limitaciones del modelo, es decir, saber concretamente el nivel de rentabilidad de los activos para que la empresa genere valor sin importar lo complejo de la organización.

\section{CONCLUSIONES}

Colombia sustenta su economía en gran medida por las mipymes, estas, a su vez, representan el $35 \%$ del PIB, así como el $80 \%$ del empleo (sin tener en cuenta la informalidad), razón por la cual este tipo de empresas deberán estar sujetas a la protección ante una competencia cada vez más agresiva que traspasa las barreras de lo económico. En términos de financiación, las mipymes realizan inversiones con recursos propios, esto conlleva una dificultad para cumplir los compromisos financieros asumidos, generando riesgos morales y volatilidad en retornos futuros de la empresa.

Por lo tanto, financiar activos con deuda representa una ventaja directa a la hora del pago de impuestos, lo que se traduce en mayores utilidades para los accionistas, siempre y cuando la política de endeudamiento sea coherente con el desempeño de la empresa; en ese sentido, a pesar de evaluar el WACC bajo distintos escenarios (utilizando el modelo obtenido), las pymes no llegaron a conseguir que dicho costo fuese igual o superior a las de las microempresas. Igualmente, las pequeñas empresas que financian parte de sus activos con deuda demostraron tener un mejor comportamiento en términos del costo promedio ponderado de capital frente a la mediana empresa, sin que eso represente que dichas diferencias sean significativas en términos estadísticos.

Respecto al modelo, es importante mostrar que no tuvo en cuenta la microempresa por su particular comportamiento frente a su estructura financiera, ya que el $100 \%$ de sus activos son financiados por el capital que aportan los accionistas, lo que representa un WACC constante sin importar sus particularidades. Es así, que el modelo obtenido, además de ser estimado por el método de mínimos cuadrados ordinarios empleando software $R$ statistics versión 4.0.0 que goza de un amplio reconocimiento por la comunidad científica, paso las pruebas de diagnóstico respectivas. Sin embargo, debido al tamaño de la muestra, no es generalizable a todo el sector metalmecánico 
nacional sino específicamente a las empresas que se ubican en el Área Metropolitana de Bucaramanga.

Además, el modelo logró identificar cuantitativamente las consecuencias que trae en el WACC el aumento de las tasas de interés por unidad porcentual; asimismo, logró cuantificar el cambio en el WACC al incrementar el porcentaje de equity en la estructura financiera. De esta manera, el modelo no solamente logró estimar cuantitativamente el WACC como consecuencia de los cambios que se producen en la estructura financiera, sino que además pudo hacer una comparación entre las mipymes del sector bajo distintos escenarios previamente establecidos. Como limitaciones y futuras líneas de investigación, se propone realizar la evaluación pertinente con otras técnicas avanzadas de pronóstico, ya que el método de MCO, en la mayoría de los casos, no se ajusta al comportamiento histórico de los datos, por lo tanto, el coeficiente de determinación ajustado no es el mejor, más aún cuando se analiza bases de datos con múltiples variables y secuencias.

\section{CONFLICTOS DE INTERÉS}

Los autores declaran que no presentan conflictos de interés financiero, profesional o personal que pueda influir de forma inapropiada en los resultados obtenidos o las interpretaciones propuestas.

\section{CONTRIBUCIÓN DE AUTORES}

Para el desarrollo de este proyecto todos los autores han realizado una contribución significativa especificada a continuación:

Ricardo Fernando Burbano-Delgado: conceptualización, diseño y desarrollo de la investigación

Youseline Garavito-Hernández: búsqueda de información, conceptualización, redacción, revisión final del manuscrito.

\section{REFERENCIAS}

Asociación Nacional de Instituciones Financieras - ANIF. (2018). La Gran Encuesta PYME. URL

Balboa, M., Martí, J., Tresierra-Tanaka, Á. (2017). Are firms accessing venture funding more financially constrained? New evidence from capital structure adjustments. The European Journal of Finance, v. 23, n. 3, 243-265. https://doi.org/10.1080/1351847X.2016.1151803

Banco de la República. (s.f.). Índice de producción real de la industria manufacturera colombiana. URL

Barrera Lievano, J. A., Parada Fonseca, S. P., Serrano Serrato, L. V. (2019). Análisis empírico de correlación entre el indicador de estructura de capital y el indicador de margen de utilidad neta en PYMEs. Revista de Métodos Cuantitativos para la Economía y la Empresa, v. 29, 99115. https://doi.org/10.46661/revmetodoscuanteconempresa.3520 
Bonilla Bonilla, Y. M. (2016). Modelo para estimar el costo de uso de capital (WACC) para las empresas PYMES del Sector Industria Caucho y Plástico de la ciudad de Bogotá [Tesis de maestría, Universidad Santo Tomás]. http://dx.doi.org/10.15332/tg.mae.2020.0741

Bruner, R., Kenneth, M. E., Robert, S. H., Robert, F. H. (1998). Best Practices in Estimating the Cost of Capital: Survey and Synthesis. Financial Practice and Education, v. 8, n. 1, 13-28. URL

Cao, J., Cui, Y. (2021). Trade Credit and Capital Structure Adjustment Speed: Evidence From Chinese Listed Firms. Review of Pacific Basin Financial Markets and Policies, v. 24, n. 1, 2150002. https://doi.org/10.1142/S0219091521500028

Castro-Silva, H. F., Rodríguez-Fonseca, F., Martínez-Chaparro, Y. A. (2019). Success factors of MSMEs in Colombia. Clío América, v. 13, n. 26, 318-327. https://doi.org/10.21676/23897848.3429

Confecámaras. (2019). Dinámica de creación de empresas en Colombia. Confecámaras.

Cornejo Díaz, R. H. (2015). Estructura de capital en mercados emergentes. Velocidad de ajuste de la estructura de capital en las empresas peruanas cotizadas en bolsa. Editorial Académica Española.

Cotner, J. S., Fletcher, H. D. (2000). Computing the Cost of Capital for privately held firm. American Business Review, v. 18, n. 2, 27-33. URL

Das, M. S. (2015). How IFRS based financial statement define the relationship between capital structure and firm's profitability: an analysis based on selected Indian companies. Pacific Business Review International, v. 8, n. 2, 31-36. URL

Departamento Administrativo Nacional de Estadística - DANE. (2018). Boletín Técnico Encuesta Anual Manufacturera EAM 2018. URL

EMIS Profesional (s.f.). Software. $\underline{U R L}$

Fernández, P., Carabias, J. M. (2006). Market Risk Premium: Historical, Expected, Required and Implied. IESE Business School, 1- 22. http://dx.doi.org/10.2139/ssrn.897676

Faraway, J. J. (2015). Linear Models with R. (2 ed.). CRC Press.

Hall, G. C., Hutchinson, P. J., Michaelas, N. (2004). Determinants of The Capital Structures of European SMEs. Journal of Business Finance \& Accounting, v. 31, n. 5-6, 711-728. https://doi.org/10.1111/i.0306-686X.2004.00554.x

Hovakimian, A., Li, G. (2011). In search of conclusive evidence: how to test for adjustment to target capital structure. Journal of Corporate Finance, v. 17, n. 1, 33-44.

https://doi.org/10.1016/j.jcorpfin.2010.07.004 
Jackova, A. (2017). The financial structure optimization-a strategic tool for competitive fight of companies. Ad Alta: Journal of Interdisciplinary Research, v. 7, n. 1. URL

Ley 590 de 2000. Por la cual se dictan disposiciones para promover el desarrollo de las micro, pequeñas y medianas empresa. (2000, 10 de julio). Congreso de la República de Colombia. No. 44.078. $\underline{\text { URL }}$

Martínez, C. E., Ledesma, J. S., Russo, A. (2014). Modelos de cálculo de las betas a aplicar en el Capital Asset Pricing Model: el caso de Argentina. Estudios Gerenciales, v. 30, n. 131, 200-208. https://doi.org/10.1016/j.estger.2014.03.002

Modigliani, F., Miller, M. H. (1958). The Cost of Capital, Corporation Finance, and the Theory of Investment. The American Economic Review, v. 48, n. 3, 261-297. URL

Modigliani, F., Miller, M. H. (1963). Corporate income taxes and the cost of capital: A correction. The American Economic Review, v. 53, n. 3, 433-443. $\underline{\text { URL }}$

Pachón Palacios, M. L. (2013). Modelo Alternativo para Calcular el Costo de los Recursos Propios. Universidad EAN. https://doi.org/10.21158/9789587562002

Parra Barrios, A. (2018). Un acercamiento al costo de capital de la empresa: naturaleza y aplicación en las decisiones financieras. Contexto, v. 7, 5-22. $\underline{\text { URL }}$

Rodríguez, A. G. (2003). La realidad de la pyme colombiana. Desafío para el desarrollo. Fundes internacional. $\underline{U R L}$

Rodríguez-Lavado, M. (2018). Bioinformática: Inteligencia Artificial para profundizar en el conocimiento de trastornos metabólicos complejos. [Trabajo de grado, Universidad Complutense de Madrid].

Ross, S. A. (1976). The Arbitraje Theory of Capital Asset Pricing. Journal of Economic Theory, v. 13, n. 3, 341-360. https://doi.org/10.1016/0022-0531(76)90046-6

Sánchez Segura, J. H. (2010). La tasa de descuento en países emergentes aplicación al caso colombiano. Revista Escuela De Administración De Negocios, v. 69, 120-134. https://doi.org/10.21158/01208160.n69.2010.520

Sharpe, W. F. (1964). Capital Asset Prices: A Theory of Market Equilibrium under conditions of risk. The Journal of Finance, v. 19, n. 3, 425-442. https://doi.org/10.1111/i.1540-6261.1964.tb02865.x

Situm, M. (2021). Determination of expected cost of equity with the CAPM: Theoretical extension using the law of error propagation. Managerial and Decision Economics, v. 42, n. 1, 77-84. https://doi.org/10.1002/mde.3214 
Stiglitz, J. (1974). On the irrelevance of corporate financial policy. The American Economic Review, v. 64, n. $6,851-866 . \underline{U R L}$

Torralba Flores, A., Pérez Paredes, A., Martínez Ángeles, D. M. (2017). Fracaso emprendedor, como experiencia de aprendizaje para empresarios del municipio de Puebla. Revista GEON (Gestión, Organizaciones y Negocios), v. 4, n. 2, 25-33. https://doi.org/10.22579/23463910.21

Tusell, F. (2011). Análisis de Regresión. Introducción Teórica y Práctica Basada en R. Universidad de Bilbao. $\underline{U R L}$

Valverde, G. R., Valverde, B. R. (2006). Colinealidad y mínimos cuadrados ponderados. Revista venezolana de análisis de coyuntura, v. 12, n. 1, 283-296.

Wojewodzki, M., Poon, W. P. H., Shen, J. (2018). The role of credit ratings on capital structure and its speed of adjustment: An international study. The European Journal of Finance, v. 24, n. 9, 735-760. https://doi.org/10.1080/1351847X.2017.1354900 